\title{
Quantum phases of attractive bosons on a Bose-Hubbard ladder with three-body constraint
}

\author{
Manpreet Singh ${ }^{1}$, Tapan Mishra ${ }^{2}$, Ramesh V. Pai ${ }^{3}$ and B. P. Das ${ }^{1}$ \\ ${ }^{1}$ Indian Institute of Astrophysics, II Block, Koramangala, Bangalore-560 034, India \\ ${ }^{2}$ Institut für Theoretische Physik, Leibniz Universität Hannover, Appelstr. 2, 30167 Hannover, Germany and \\ 3 Department of Physics, Goa University, Taleigao Plateau, Goa 403 206, India
}

(Dated: August 2, 2021)

\begin{abstract}
We obtain the complete quantum phase diagram of bosons on a two-leg ladder in the presence of attractive onsite and repulsive interchain nearest neighbor interactions by imposing the onsite three body constraint. We find three distinct phases, namely, the atomic superfluid(ASF), dimer superfluid (DSF) and the dimer rung insulator (DRI). In the absence of the interchain nearest neighbor repulsion, the system exhibits a transition from the ASF to the DSF phase with increasing onsite attraction. However, the presence of the interchain nearest neighbor repulsion stabilizes a gapped DRI phase, which is flanked by the DSF phase. We also obtain the phase diagram of the system for different values of the interchain nearest neighbor interaction. By evaluating different order parameters, we obtain the complete phase diagram and the properties of the phase transitions using the self consistent cluster mean field theory.

PACS numbers: 75.40.Gb, 67.85.-d, 71.27.+a
\end{abstract}

\section{INTRODUCTION}

Systems of ultracold bosonic atoms in optical lattices have acquired a lot of importance from several areas of physics, in particular in the field of condensed matter and AMO physics [1]. The flexibility in controlling the system parameters by tuning the laser intensity and the technique of Feshbach resonance, makes them a versatile tool for simulating many interesting physical systems. This results in the observation of various quantum phase transitions which would otherwise be very difficult to study using solid-state systems. After the first prediction 2] and observation [3] of the superfluid (SF)-Mott insulator (MI) transition in the Bose-Hubbard model in an optical lattice, enormous progress has been made in the last decade or so. It has been proposed that a system of polar gases in optical lattices can give rise to a crystalline phase [4] due to the long range van der Waal type interaction. Under certain conditions, it is possible to stabilize the exotic supersolid phase [5 13]. The observation of chromium Bose-Einstein condensate (BEC) [14, 15] followed by the realization of quantum gases in other highly-magnetic species, including dysprosium Bose and Fermi gases [16] and erbium condensate [17], experiments on polar molecules such as KRb [18] and the Rydberg gases [19] have opened up possibilities for manipulating the off-site interactions in optical lattices.

Low dimensional systems have been studied widely in the past few decades In particular, $1 D$ or quasi- $1 D$ systems are of very special interest because interactions play a crucial role in realizing novel phases [20, 21]. Research on ultracold atoms in optical lattices offers a unique platform to engineer various lattice models which can mimic physical phenomena [20]. Quasi-1D systems such as ladders have been of special interest to understand the phenomenon of high-temperature superconductivity, spin-gapped metallic state 22 24] etc. The extra cou- pling between the legs of the ladder makes these systems unique, as a result of which, the quantum phase transitions are influenced substantially even in a simple model like the Bose-Hubbard ladder [25 27]. Also, the effect of kinetic frustration along with various interactions can lead to interesting new phases in ladder systems [28 36 , which are not possible in one dimensional lattice systems. In recent years, it has been shown that novel quantum phases such as a trimer liquid and the devil's staircase can arise from atoms and molecules possessing long range interactions [37, 38]. In the experiments ladder models can be realized in the systems of optical lattices [39 42].

Earlier studies have shown that an ultracold bosonic gas in a lattice with attractive interactions undergoes a transition from an atomic superfluid (ASF) to the dimer superfluid (DSF) phase when the atoms are subjected to the onsite three body constraint [43]. This phenomenon was first predicted in the context of an atomic Bose gas in the continuum with Feshbach resonance [45]. This prediction suggests that the bosons can pair up to form the DSF phase when the attraction between them is sufficiently large. This transition was predicted to be Ising like at the commensurate filling and first order at other fillings. Detailed investigations on this model have been made on a square lattice in the recent past [46, 47] to obtain the ground state phase diagram. The effect of nearest neighbor interaction on a square lattice has predicted a dimer checker board solid phase [48]. Bosons with two-body onsite attractive interactions in optical lattices that are subjected to the onsite three- or fourbody constraint can form dimer and density wave phases as well [43, 49, 50].

In this paper, we focus on a system of ultracold bosonic atoms possessing long-range interactions along the rungs of a two leg ladder as shown in Fig. 11 This system can be realised by using dipolar bosons and polarizing them at the magic angle $\phi$ with reference to the plane 


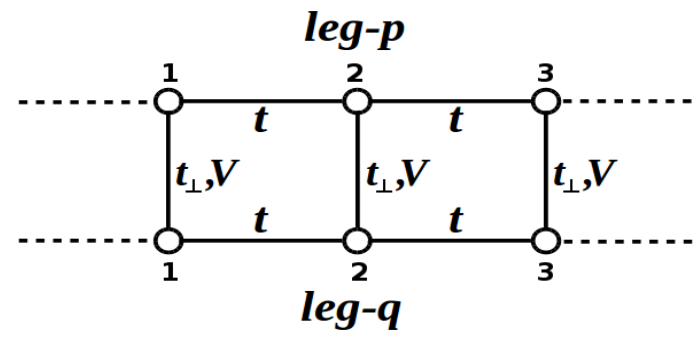

FIG. 1. A small section of the ladder model is shown and the cluster contains six sites. Dashed lines represent bonds between decoupled sites, circles represent lattice sites.

of the ladder, and perpendicular to the rungs of the ladder. The dipole-dipole interaction energy is proportional too $\left(1-3 \cos ^{2} \phi\right)$. Therefore if the angle $\phi$ is chosen such that the condition $\cos ^{2} \phi=1 / 3$ is satisfied, the dipoledipole interaction energy along the leg becomes zero but remains finite along the rungs. Taking the onsite interactions to be attractive for such a system and imposing the three body constraint, we study its ground state phase diagram. In addition, we also study the quantum phases of this model in the case of hard core bosons to validate our predictions. We use the self consistent cluster mean-field theory (CMFT) to determine the various order parameters to obtain the different quantum phase transitions in this model.

The remaining part of this paper is organized as follows. In Sec. II we give details of the model and the method used in our calculation. Section III is devoted to our results. Sec. IV contains concluding remarks.

\section{MODEL AND METHOD}

The effective many-body lattice model which describes the problem mentioned above is given by

$$
\begin{aligned}
H= & -t \sum_{\alpha, i}\left(a_{\alpha, i}^{\dagger} a_{\alpha, i+1}+H . c .\right)-t_{\perp} \sum_{i}\left(a_{p, i}^{\dagger} a_{q, i}+H . c .\right) \\
& +\frac{U}{2} \sum_{\alpha, i} n_{\alpha, i}\left(n_{\alpha, i}-1\right)+V \sum_{i} n_{p, i} n_{q, i}-\sum_{i} \mu_{\alpha, i} n_{\alpha, i}
\end{aligned}
$$

where $a_{\alpha, i}{ }^{\dagger}\left(a_{\alpha, i}\right)$ is the bosonic creation (annihilation) operator at the site $i$ of leg $-\alpha(=p, q)$. $t$ is the hopping amplitude between the nearest neighbor sites along the legs of the ladder and $t_{\perp}$ is the hopping along the rungs of the ladder. $n_{\alpha, i}=a_{\alpha, i}^{\dagger} a_{\alpha, i}$ is the number operator at site $(\alpha, i) . \quad U$ and $V$ represent the on-site inter-atomic two-body and nearest-neighbor interactions respectively. The chemical potential is represented by $\mu$. In this work we assume the three body constraint, i.e. $\left(a^{\dagger}\right)^{3}|0\rangle=0$ and the value of $U$ is negative. We also assume that the ladder is arranged in such a way that the nearest neighbor repulsion $V$ is only present along the rungs of the ladder.

The ground state properties of this model can be studied quite accurately by using powerful numerical methods such as the density matrix renormalization group method (DMRG) [51, 52] or the quantum monte carlo (QMC) methods. However, in order to qualitatively understand the quantum phase transitions exhibited in this model, we use the self consistent CMFT method. This method is capable of capturing the relevant physics that arises due to quantum correlations, which was not always possible to achieve in the conventional single site mean-field theory decoupling approximation [53 58$]$. The CMFT can account for non-local interaction more accurately by retaining them in the exact form. For the regular SF-MI transition the estimation of the crtical point imporves when CMFT is used and approaches the value obtained by the methods like QMC and DMRG [60]. In this method a cluster of sites is treated exactly and the meanfield approximation is for the coupling which connects the cluster with rest of the lattice. The accuracy of the calculation depends on the size of the cluster considered. As the cluster size increases, the number of decoupled bond reduces and the Hamiltonian approaches the exact form. In one of its very first applications this method was used to study the phase diagram of a one-dimensional optical superlattice [59]. In recent years it has been used to study various other models where the results are found to be in good agreement with those obtained using exact numerical methods 60 63].

We consider six sites cluster as shown in Fig 1. Then the model given in Eq. (11) can be written as

$$
H=\sum_{k} H_{c}^{k}
$$

where summation over $k$ is over all the clusters and $H_{c}^{k}$ is the Hamiltonian for $k^{\text {th }}$ cluster which can be written as (dropping $k$ since all the clusters are identical)

$$
H_{c}=H_{e}+H_{d}
$$

where $H_{e}$ is the exact Hamiltonian of the cluster and $H_{d}$ is the decoupled hopping term to the nearest neighbor cluster. Using the mean-field decoupling approximation one can make the following substitution since $i$ and $j$ are adjacent sites in the nearest neighbor clusters,

$$
a_{i}^{\dagger} a_{j} \simeq\left\langle a_{i}^{\dagger}\right\rangle a_{j}+a_{i}^{\dagger}\left\langle a_{j}\right\rangle-\left\langle a_{i}^{\dagger}\right\rangle\left\langle a_{j}\right\rangle
$$

in the hopping term in Eq.(1) and introduce the superfluid order parameter

$$
\phi_{i} \equiv\left\langle a_{i}^{\dagger}\right\rangle \equiv\left\langle a_{i}\right\rangle
$$

to obtain the following Hamiltonian for the decoupled part,

$$
H_{d}=-t \sum_{\alpha=\{p, q\}} \sum_{\substack{i, i^{\prime}=1,3 \\ i \neq i^{\prime}}}\left[\phi_{\alpha, i}\left(a_{\alpha, i^{\prime}}{ }^{\dagger}+a_{\alpha, i^{\prime}}\right)+\phi_{\alpha, i} \phi_{\alpha, i^{\prime}}\right]
$$


The exact Hamiltonian, $H_{e}$ is given by

$$
\begin{aligned}
H_{e}= & -t \sum_{\substack{\alpha=\{p, q\} \\
i=\{1,2\}}}\left(a_{\alpha, i}^{\dagger} a_{\alpha, i+1}+H . c .\right) \\
& -t \sum_{\perp}\left(a_{p, i}^{\dagger} a_{q, i}+H . c .\right) \\
& +\sum_{\substack{\alpha=\{p, q\} \\
i=\{1,2,3\}}}\left[\frac{U}{2} n_{\alpha, i}\left(n_{\alpha, i}-1\right)+V n_{p, i} n_{q, i}-\mu_{\alpha, i} n_{\alpha, i}\right]
\end{aligned}
$$

We set the energy scale by choosing $t=1$, as a result, all the physical parameters considered are dimensionless. We choose to work in the occupation number basis and construct the Hamiltonian matrix using the expression given by $H_{c}$. This matrix is then diagonalized self-consistently to obtain the ground state of the system. The ground state so obtained can then be used to calculate the necessary expectation values.

\section{RESULTS AND DISCUSSION}

In this section we report our findings and anlayze the results of our work. Before presenting the main results, we validate our method; i.e. the CMFT by studying an already known phase diagram using other exact methods. It has been predicted that in the case of hardcore bosons $(U=\infty)$ on a ladder, the system exhibits a rung Mott insulator (RMI) phase due to the competition between the intra-chain and inter-chain couplings, $t$ and $t_{\perp}$ respectively [64, 65]. When the value of $t_{\perp}$ is large compared to $t$, the atoms can only hop within the rungs of the ladder, which results in the system being gapped. We have investigated this model using our CMFT approach and obtain the phase diagram shown in Fig 2 This phase diagram is in a very good qualitative agreement with the results presented in Ref. 65]. We have done the calculations using $4-, 6-, 8-$ and $10-$ sites clusters. The $\mathrm{RMI}(1 / 2)-\mathrm{SF}$ critical transition point found from each of these calculations are then scaled to the thermodynamic limit. This gives an estimate of $\left(t_{\perp} / t\right)_{c} \sim 2.087$.

We now turn to the results of our work. Recent numerical and analytical works on the model given in Eq.(1), in the absence of the nearest neighbor interactions, have predicted the existence of a transition from the ASF phase to the DSF phase in one and two dimensions [43, 46 49]. Apart from this, two trivial gapped (insulating) phases, $\operatorname{MI}(0)$ at $\rho=0$ and $\operatorname{MI}(2)$ at $\rho=2$, are also present. It has been predicted for a two dimensional square lattice that (i) the MI to DSF transition is always continuous, (ii) there exists a first order transition from the $\mathrm{MI}(0)$ to ASF phase and (iii) there exists a tricritical point along the ASF-MI(2) transition boundary [46]. In the presence of the nearest neighbor interaction $V$, the region of the first order phase boundary shrinks as $V$ is increased. A dimer checker board solid (DCS) appears when $V \neq 0$ [48.

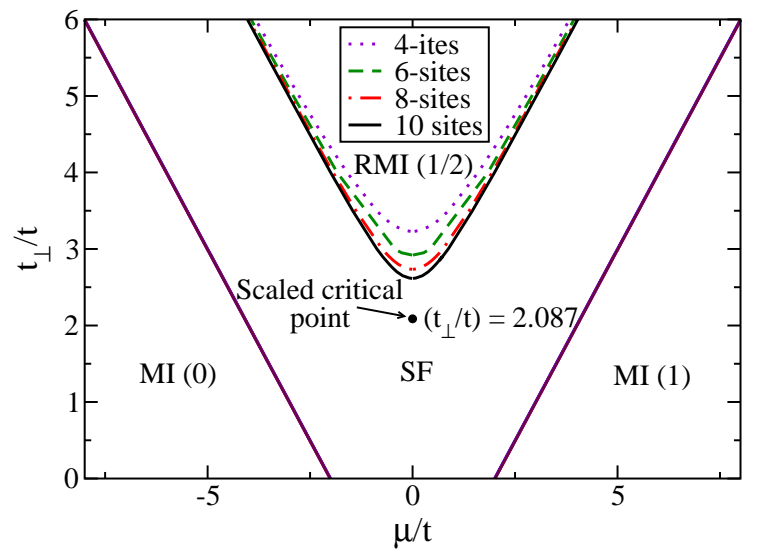

FIG. 2. (Color online) Phase diagram for hard-core bosons in absence of $V$ for different cluster sizes. The cluster sizes are indicated in the legend. The scaled critical point for $\mathrm{RMI}(1 / 2)-\mathrm{SF}$ transition is represented by a black dot.

As stated earlier, the system we consider here is a two leg ladder and the nearest neighbor interaction is allowed only along the rungs of the ladder. We have considered three different cases: $V=0.0,0.5$ and 1.0 to demonstrate the salient features of phase diagrams for these three cases. When $V=0.0$, the system exhibits a phase diagram qualitatively similar to that obtained for the square lattice case as shown in Fig, 4. This phase diagram consists of four phases, the MI(0), MI(2), ASF and DSF phases. To obtain this in our CMFT approach, we use the superfluid order parameter $\phi$ and the density $\rho$ as the order parameters. In the ASF phase, the atomic superfluid density $\rho_{s}=\phi^{2}=\left\langle\left(a^{\dagger}\right)\right\rangle^{2}$ is finite and is zero for the MI phases. In other words the ASF phase is gapless and compressible and the MI phases are gapped. In order to obtain the phase boundary between different phases we plot the density $\rho$ (solid line (red curve)) and superfluid density $\rho_{s}$ (circles (green curve)) as a function of the chemical potential $\mu$ for different values of $U$ in Fig 3 where it can be seen that the $\rho$ versus $\mu$ plot (solid (red) curves) has two plateaus corresponding to the gapped $\operatorname{MI}(0)$ and $\operatorname{MI}(2)$ phases at $\rho=0$ and $\rho=2$ respec-

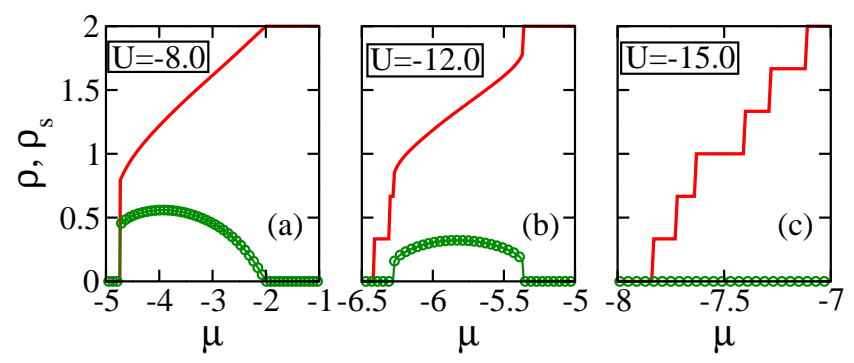

FIG. 3. (Color online) $\rho, \rho_{s}-\mu$ plot for (a) $U=-8.0$, (b) $U=-12.0$ and (c) $U=-15.0$ for $V=0.0$. Solid (red) curves represent average density $\rho$ and circles (green curves) represent average superfluid density $\rho_{s}$ in the cluster. 


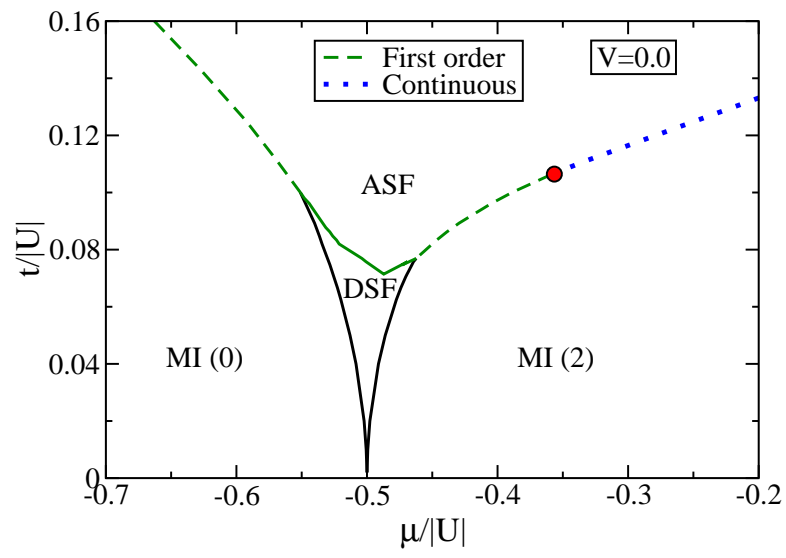

FIG. 4. (Color online) Phase diagram for $U<0$ and $V=0.0$. Green (dashed) line and blue (dotted) line indicate first and second-order transitions, respectively. The first- to secondorder change on the phase boundary is marked by a red circle.

tively. However, at intermediate densities the ASF and DSF phases appear. When $U$ is small, say -8.0 , a region exists where $\rho_{s}$ is finite, circles(green curve) in Fig [3(a) and the value of $\rho$ (solid(red) curves) increases suddenly from zero to some finite value and then increases continuously till it saturates at two. This region in the parameter space is the ASF phase since the superfluid density $\rho_{s}$ remains finite. The sudden jump in the values of $\rho$ and $\rho_{s}$ from zero to finite value suggests that the transition from the MI(0) to ASF phase is first order which will be discussed in more detail later. When $U$ becomes more negative and the value of $\mu$ is small, the attraction between particles favors dimer formation which hop around to form the DSF phase. In the framework of our CMFT approach, it is difficult to predict the dimer phases directly from the calculation of the DSF order parameter. However, if on increasing the value of the chemical potential, the system density increases in steps of two atoms,

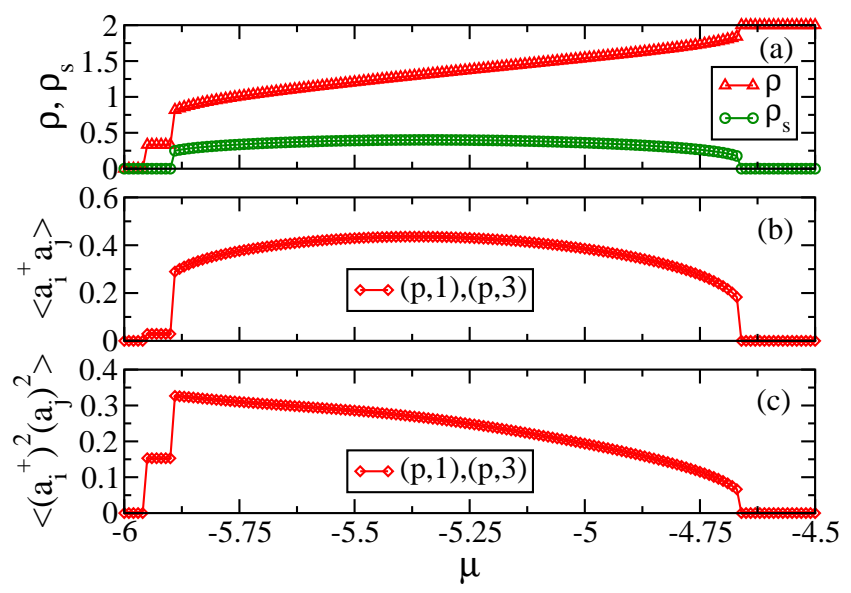

FIG. 5. (Color online) $U=-11.0, V=0.0$. (a) $\rho, \rho_{s}-\mu$ plot (b)single-particle tunneling and, (c)pair tunneling amplitude between sites. then we conclude that it has entered the DSF phase. This shows that the system consists of only dimers and they behave as single entities. When the chemical potential is increased, for small values of $\mu$ as seen in the Fig 3 (b) for $U=-12.0$, the density of the system increases only if the chemical potential increases to accommodate two bosons or one dimer. However, for higher values of the chemical potential, the density increases in a continuous manner with the chemical potential, because the kinetic energy dominates in this situation and the system behaves like an atomic superfluid. At this value of $U$, a first order type transition from the ASF to the MI(2) phase takes place, which can be seen from the sharp jump in the density at the chemical potential close to the MI(2) plateau. When $U$ is highly attractive, say $U=-15.0$, all the particles form dimers and the system is fully in the DSF phase. The particle density then increases in steps of two particles till it reaches the MI(2) state. This behavior can be seen in the Fig 3(c). Since in this calculation we have considered a cluster consisting of 6 sites, we get jumps in the density when it reaches the values $1 / 3,2 / 3,1,4 / 3,5 / 3$ and 2 i.e. when the total number of bosons in the cluster is equal to, respectively, 2, 4, 6, 8, 10 and 12. By locating the transition points from the $\rho-\mu$ curves we obtain various phases and the phase diagram as shown in Fig.4. Although the DSF to ASF phase transition shows a first-order type behavior in the $\rho, \rho_{s}-\mu$ plots, it is actually predicted to be of Ising type at unit filling in other models in earlier works 43 . In our CMFT approach it is difficult to predict the nature of this transition. The small plateaus we obtain in $\rho-\mu$ curves in the DSF region are artifacts of the finite size of the system we have used in our work. We expect these plateaus to become smaller and gradually disappear as the system size is increased. In fact, the disappearance of such plateaus has been shown in a comparative study of a system of hardcore bosons using the exact-diagonalization and quantum Monte Carlo methods [4].

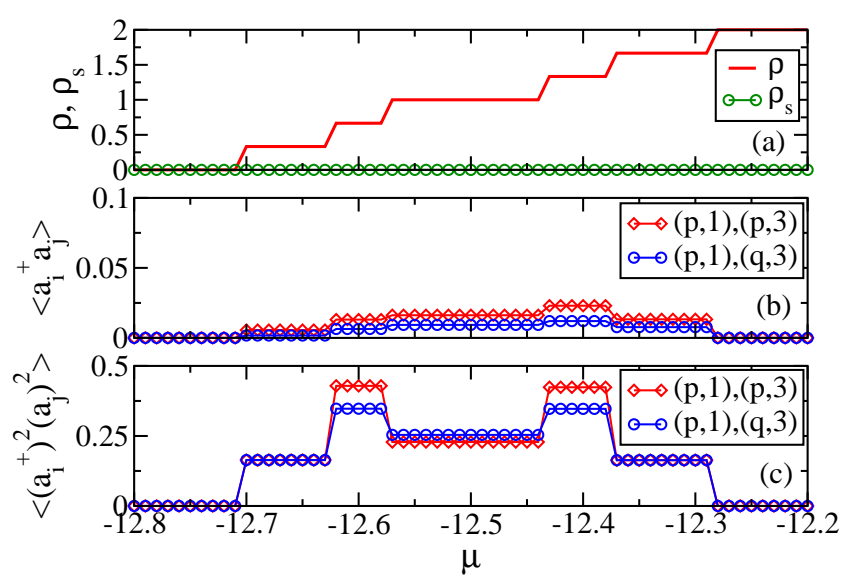

FIG. 6. (Color online) $U=-25.0, V=0.0$. (a) $\rho, \rho_{s}-\mu$ plot (b)single-particle tunneling and, (c)pair tunneling amplitude between sites. 


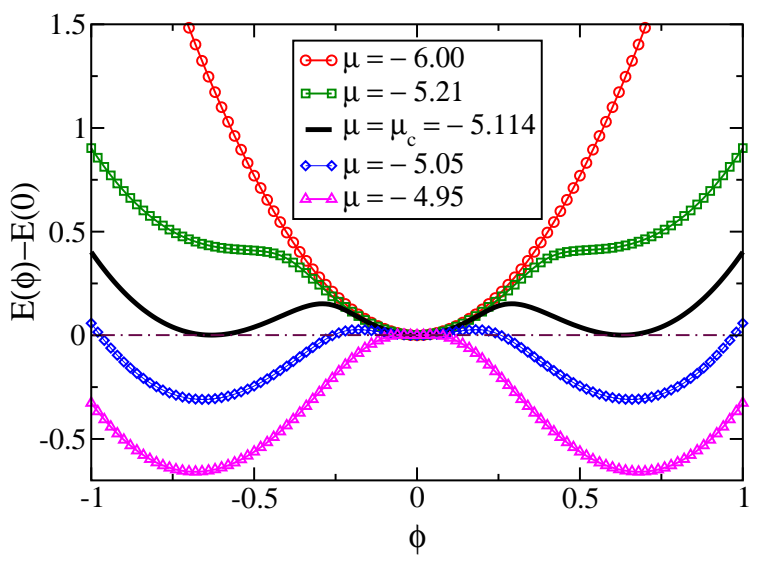

FIG. 7. (Color online) $\mathrm{E}(\phi)-\mathrm{E}(0)$ versus order parameter $(\phi)$ plot for $U=-9.0, V=0.0$. From top to bottom, $\mu=-6.0,-5.21,-5.114\left(\mu_{c}\right),-5.05$ and -4.95 for $\mathrm{MI}(0)$ ASF transition across the left most boundary in Fig 4

Another signature of the dimer formation can be inferred by comparing the single-particle tunneling and the paired-tunneling amplitudes. As an example we have plotted the above quantities for $U=-11.0$ and $U=-25.0$ in Fig 5 and Fig 6 respectively. The quantity $\left\langle a_{i}^{\dagger} a_{j}\right\rangle$ is the tunneling amplitude for a single boson and $\left\langle a_{i}{ }^{2} a_{j}{ }^{2}\right\rangle$ is the tunneling amplitude for a pair of bosons between the sites $i$ and $j$. The pair of sites $i, j$ between which tunnelings are considered are given in the legends of the respective plots. For $U=-11.0$, DSF exists only for a small region of $\mu$ values, around $\mu=-5.9$, while the ASF phase dominates the rest of the region, as shown in Fig[5] The paired-tunneling amplitude dominates over the single boson tunneling in the DSF phase and as expected both have finite values in the ASF phase. When the DSF phase dominates, as in the case for $U=-25.0$, paired-tunneling amplitude remains constant as $|i-j|$ increases while the single boson tunneling decreases to zero, as shown in Fig. [6. These features confirm our earlier conclusion that we do not have the ASF phase for higher values of $|U|$ as shown in Fig 4 . The order of the phase transition between $\mathrm{MI}(0)$ to $\mathrm{ASF}$ and $\mathrm{ASF}$ to $\mathrm{MI}(2)$ can be obtained by observing the ground state energy of the system around the critical point on the common phase boundary. We plot $E(\phi)-E(\phi=0)$ as a function of the superfluid order parameter $\phi$ for the $\mu$ values at and around the critical point. One such plot for $U=-9.0, V=0.0$ is given in Fig 7 This point lies on the phase boundary between $\mathrm{MI}(0)$ and the ASF phases (Fig(4). When $\mu<-5.114$ the system is in the MI(0) phase and we obtain a single minimum, but as $\mu$ increases and approaches the critical point $\left(\mu_{c}=-5.114\right)$, two more minima start appearing. At the exact critical point all the three minima become degenerate. A single minimum indicates a unique solution which corresponds to the MI phase and the three degenerate minima indicate the three of the possible solutions of the infinitely degenerate SF phase. This is an indicator of a first-order

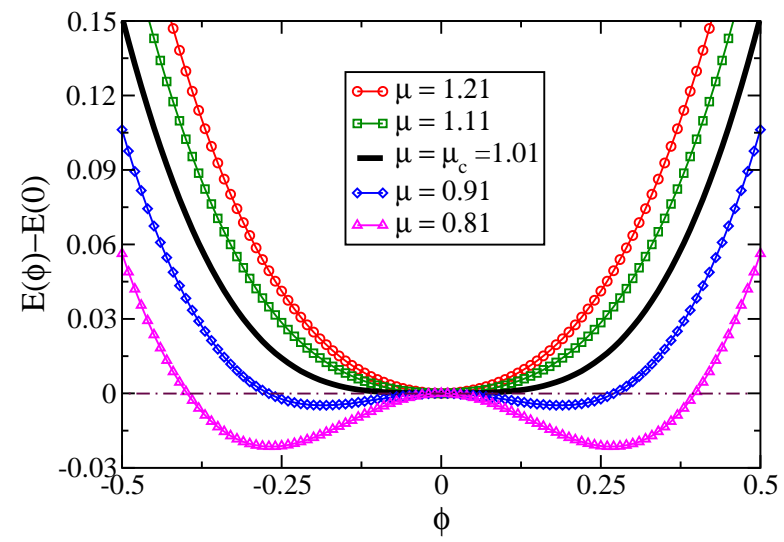

FIG. 8. (Color online) $\mathrm{E}(\phi)-\mathrm{E}(0)$ vs. order parameter $(\phi)$ plot for $U=-5.0$. From top to bottom, $\mu=$ $1.21,1.11,1.01\left(\mu_{c}\right), 0.91$ and 0.81 for $\mathrm{ASF}-\mathrm{MI}(2)$ transition across the right most boundary in Fig 4

transition.

We pick one other point at $U=-5.0, V=0.0$ on the phase boundary between ASF and $\mathrm{MI}(2)$ phases and repeat the above procedure to find out the order of transition. The corresponding plot is shown in Fig 8 . We can see that there are only two minima merging into a single minimum at this point. Therefore this is a second-order transition. We repeat these calculations for several values of $U$ around which there are sudden jumps in $\rho, \rho_{s}-\mu$ plots. For $V=0.0$ we find that the nature of the SFMI(2) transition changes from first-order to second-order at $U \sim-9.4$. This point is marked by a red circle in Fig 4, which is a tricritical point.

Now we discuss our findings by considering the effect of the nearest neighbor interaction $V$. As mentioned before, we consider the nearest neighbor interaction only along the rungs of the ladder such that the system does not break any translational symmetry by forming a density wave order. In such a situation, the effect of a small value of $V$ is dramatic when $U$ is highly attractive. We investigate the system for two different values of $V$ equal to 0.5 and 1.0. In both the cases we study the phase diagram by increasing the magnitude of $U$ and making it more attractive. When $U$ is slightly negative the system exhibits the ASF phase for densities intermediate between 0 and 2. However, when the value of $U$ is sufficient to form dimers and at $\rho=1$, the small value of $V$ tries to prevent two dimers to sit on a single rung. However, it cannot restrict the dimer to hop within the sites of a rung which is governed by the kinetic term $t_{\perp}$. Hence the dimers are localized on the rungs of the ladder creating a singlet on each rung. This phase exhibits a finite single particle gap, and vanishing superfluid order parameter. This phase can be called as the dimer rung insulator (DRI). However, for the density range $0<\rho<1$ and $1<\rho<2$, the system remains in the DSF phase. In order to obtain the phase diagram we analyze the plots of $\rho$ and $\rho_{s}$ as a function of $\mu$ and is given for $V=0.5$ in Fig 9 It is 

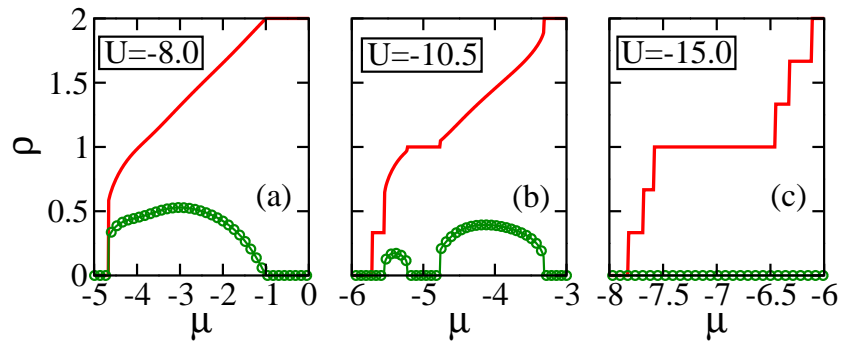

FIG. 9. (Color online) $\rho, \rho_{s}-\mu$ plot for (a) $U=-8.0$, (b) $U=$ -10.5 and (c) $U=-15.0$ for $V=0.5$. Solid (red) curves represent average density, solid-circle (green) curves represent average superfluid density in the cluster.

evident that when $U=-8.0$, the effect of $V$ is not visible as shown in Fig 9(a). However, Fig 9(b) shows that for $U=-10.5$ there appears a plateau at $\rho=1 / 3$ and $\rho=1$. The length of the $\rho=1 / 3$ plateau increases slightly but $\rho=1$ plateau increases considerably as $|U|$ increases, as shown in Fig 9(c). At the $\rho=1$ plateau region the value of $\rho_{s}$ is zero which reflects that the DRI phase is gapped. By picking the boundary points from the $\rho$ versus $\mu$ curve we obtain the phase diagram as shown in Fig 10. When the value of $V=1.0$, the DRI phase gets enlarged as shown in Fig [1] Changes in the phase boundaries with a change in cluster size are also indicated in Fig [1] A scaling of ASF-DRI critical point with 4-,6- and 8-sites clusters gives an estimate of $U_{c} \sim-7.92$ in the thermodynamic limit. The order of the phase transitions, like before, are also obtained by simultaneously observing the sharp jump in the corresponding $\rho-\mu$ plots and the quantity $E(\phi)-E(0)$. The position of the tricritical point shifts to the higher values of $\mu /|U|$ as the value of $V$ increases. This phenomena was also predicted before in a similar model for a square lattice [48]. The plateau at $\rho=1$ also appears in the DSF phase. In order to dis-

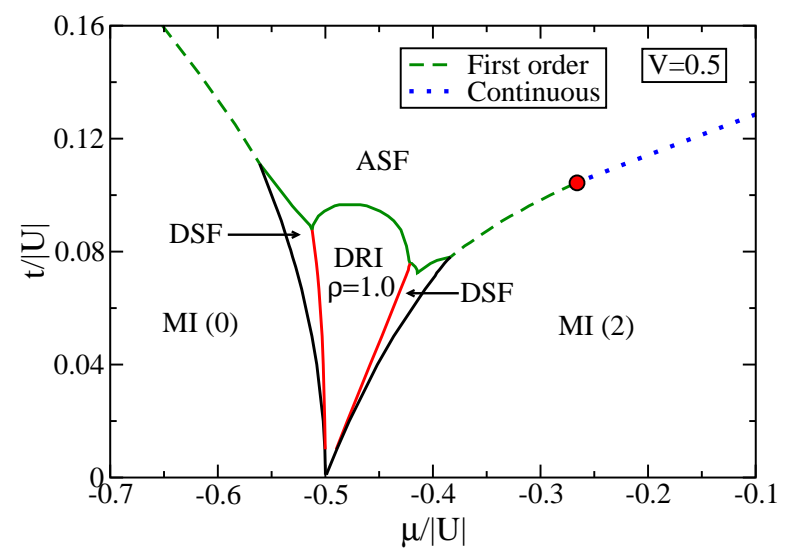

FIG. 10. (Color online) Phase diagram for $U<0$ and $V=0.5$ showing different phases. Green (dashed) line and blue (dotted) line indicate first and second-order transitions, respectively. The first- to second-order change on the phase boundary is marked by a red dot.

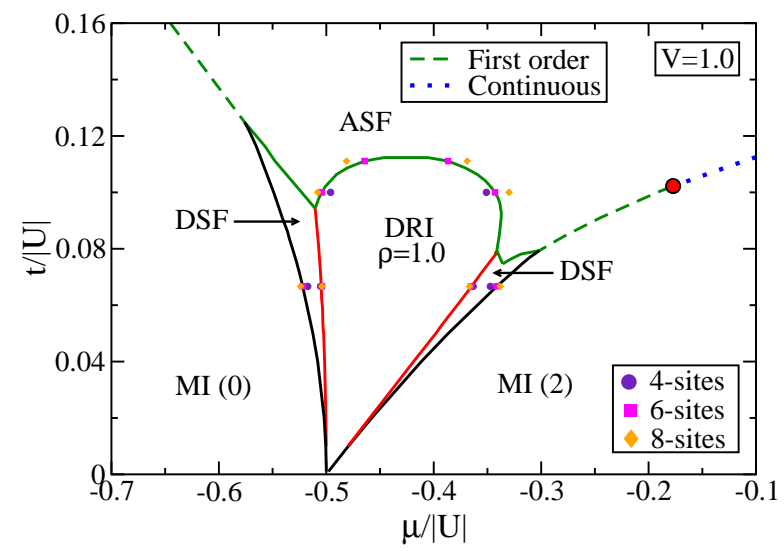

FIG. 11. (Color online) Phase diagram for $U<0$ and $V=1.0$ showing different phases. Green (dashed) line and blue (dotted) line indicate first and second-order transitions, respectively. The first- to second-order change on the phase boundary is marked by a red dot. The change in phase boundaries with cluster size is also indicated at $U=-9.0,-10.0$ and -15.0. For the 4-sites cluster, the DRI lobe does not extend beyond $U=-9.12$.

tinguish between the DSF and DRI phases, we plot the single dimer correlation function (paired-tunneling amplitudes) along the rung and the leg of the ladder. When the system is in the DSF phase, this correlation function is finite both along the rungs and the legs. However, in the DRI phase, it is large on the rungs compared to the legs. As the value of $V$ increases, they tend to zero along the legs whereas they tend to one along the rungs as shown in Fig 12 In Fig 12(a) we plot the $\rho, \rho_{s}-\mu$ plot for $U=-25.0$ and $V=1.0$ for comparison. In Fig[12(b) and (c) we plot the single particle and dimer correlations, respectively. At this density each rung has one dimer and is in a superposition of $|0, D\rangle$ and $|D, 0\rangle$ states, where $D$ stands for a dimer. It becomes energetically unfavorable for a dimer to hop from one rung to the another as the

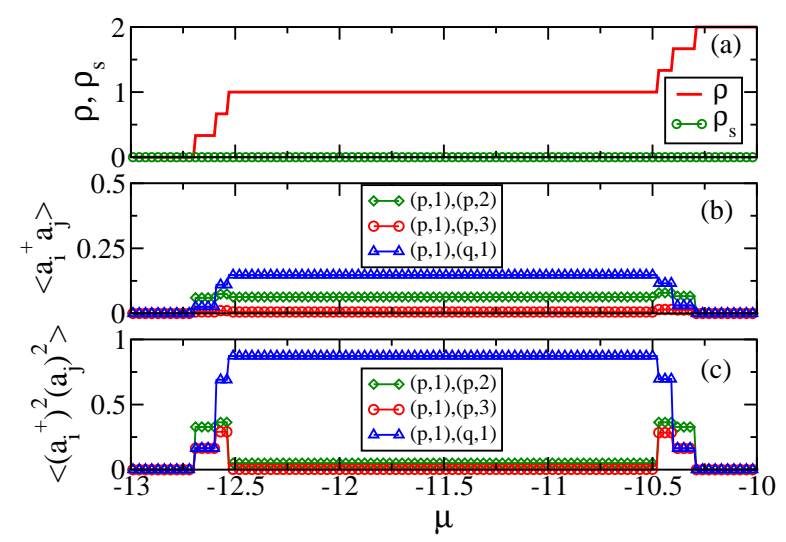

FIG. 12. (Color online) $U=-25.0, V=1.0$. (a) $\rho, \rho_{s}-\mu$ plot (b)single particle tunneling amplitude between sites (c)pair tunneling amplitude between sites. 

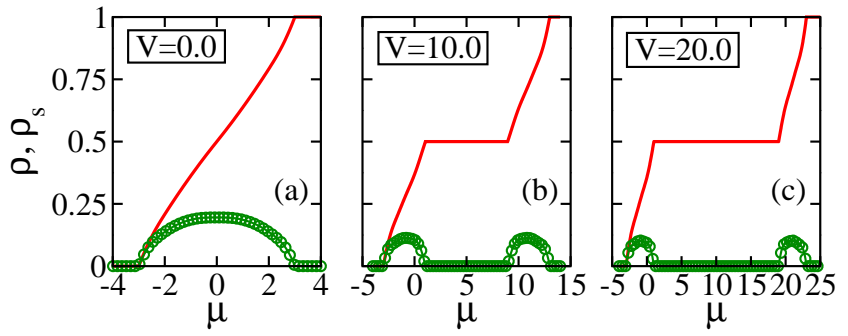

FIG. 13. (Color online) $\rho, \rho_{s}-\mu$ plot for HC bosons for (a) $V=$ 0.0 (b) $V=10.0$ and (c) $V=20.0$.

presence of $V$ will tend to increase the energy. As a result dimers get confined to their respective rungs. Therefore, we argue that the phase which appears at $\rho=1$ in the presence of $V$ is the DRI phase. The stability of the DRI phase in the thermodynamic limit is difficult to predict using the CMFT, as it takes into account only a limited number of sites in a cluster. One needs to perform rigorous numerical calculations to understand this phase in more detail.

In order to further clarify the existence of the DRI phase, we study a model of hardcore bosons on a two leg ladder with the nearest neighbor interactions acting along the rungs. This model at half filling is similar to the model discussed above in the limit of large attractive $U$ at unit filling when all the atoms have formed dimers. In the previous case, because of the three body constraint the dimers behaved like hardcore bosons. Therefore, it is indeed possible to get the rung insulator (RI) phase in a similar model of hardcore bosons. The phase-diagram for hard-core bosons at $V=0.0$ is trivial and there are only SF and MI phases. For low values of $\mu$ the system is in the $\rho=0.0 \mathrm{MI}$ phase. As $\mu$ is increased, the density of the system increases continuously, it enters the SF phase and finally ends up in the $\rho=1.0 \mathrm{MI}$ phase. However, by switching on the value of $V$, we obtain a plateau at $\rho=0.5$ which gets enlarged as the value of $V$ increases,

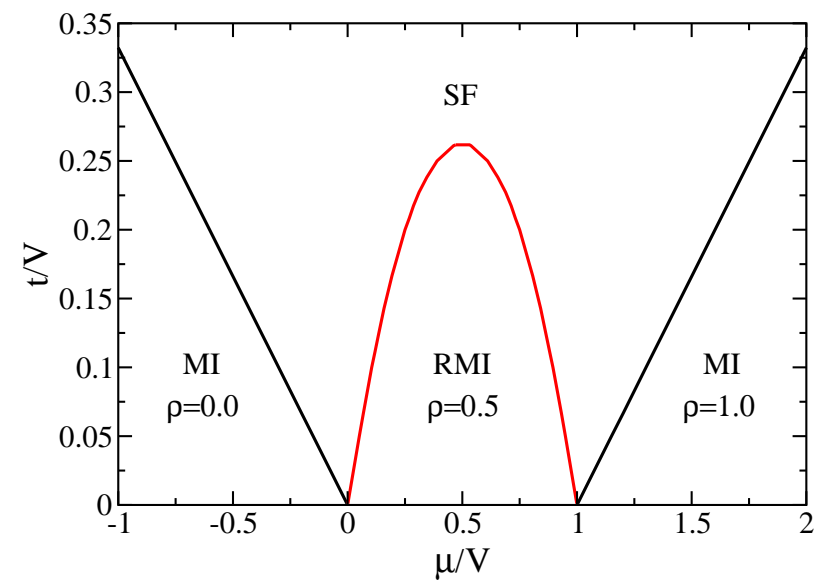

FIG. 14. (Color online) Phase diagram for hard-core bosons in the presence of interchain nearest neighbour interaction $V$.
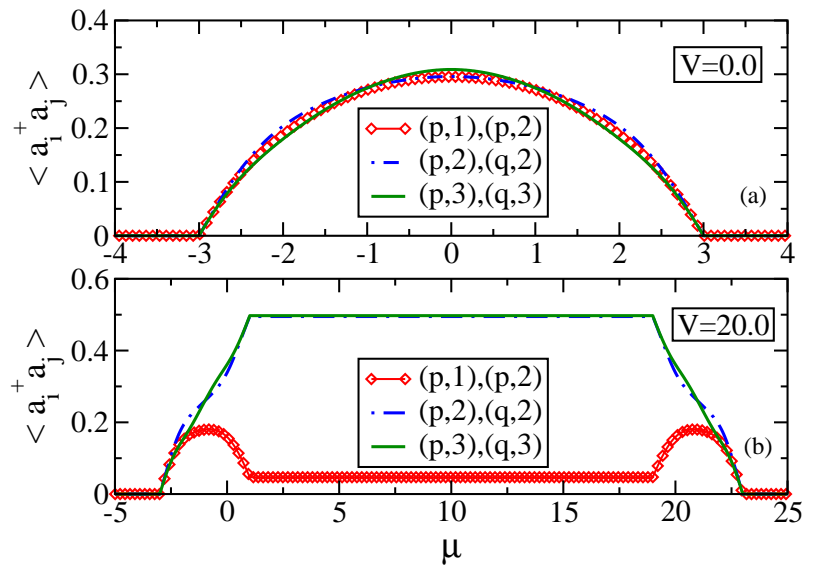

FIG. 15. (Color online) Single particle tunneling amplitudes for hardcore bosons at (a) $V=0.0$ and, (b) $V=20.0$.

as shown in Fig 13. The argument here is that, at $\rho=$ 0.5 and at finite $V$ the favorable ground state is when each rung of the ladder has only one hardcore boson. In such a situation a singlet of hardcore bosons is formed along the rungs, which is a rung Mott insulator phase as discussed before. We obtain the phase diagram for this model which is shown in Fig 14. The presence of the RI phase can be further confirmed by comparing Fig 15(a) and Fig. 15(b). We can see that in the former case when $V=0$, the tunneling amplitudes between all the sites are almost the same for all values of $\mu$ but in the latter cases when $V \neq 0$ they become different. For $V \neq 0$, at $\rho=0.5$ the tunneling amplitude of bosons within the same leg decreases and within a rung it increases.

\section{CONCLUSIONS}

We have studied the phases and the phase transitions in an attractive Bose-Hubbard model on a two leg ladder in the presence of the three body constraint. We obtain the ground state phase diagram of this model by using the self consistent cluster mean-field theory. By calculating various physical parameters of interest, we find that there exists a transition from the ASF to the DSF phases when the density of the system varies from zero to two. When the density is zero and two we obtain two gapped phases such as $\mathrm{MI}(0)$ and $\mathrm{MI}(2)$. By introducing nearest neighbor interactions between the particles sitting in the two sites of a rung, we obtain the dimer rung insulator(DRI) phase at unit filling. The DRI phase is gapped in which the particle motion is confined within the rungs of the ladder. This phase appears in the middle of the DSF phase which gets enhanced as the value of the nearest neighbor interaction increases. We also find that the $\mathrm{MI}(0)$-ASF transition boundary is first order. However, the ASF-MI(2) phase boundary is continuous for small values of $|U|$ and becomes first order when $|U|$ is large through a tricritical point. This point shifts to- 
wards the smaller values of $|U|$ as the value of $V$ increases. We also complement our prediction of the DRI phase by studying a system of hardcore bosons on a two leg ladder with nearest neighbor repulsions only along the rung. To check the stability of the phases and scaling of the critical points we have done the calculations using 4, 6, 8 and 10 site clusters for the hardcore bosons. For the soft-core bosons with the three-body constraint we have done calculations upto 8-sites keeping $V$ fixed at 1.0. In the case of hard-core bosons on a ladder we find that phase diagram improves with the increase in cluster size and the RI-SF critical point approaches the value as obtained from DMRG and QMC calculations. In the case of softcore bosons with the three-body constraint we find that overall the phase diagram remains the same qualitatively and there are only small changes in the phase boundaries with the change in cluster size.

\section{ACKNOWLEDGMENT}

We would like to acknowledge L. Santos, H. Weimer, M. Dalmonte, B. Laburthe-Tolra, S. Mukerjee, A. Nunnenkamp, and D. Huerga for scientific discussions. The computational results reported in this work were performed on the high performance computing facilities of IIA, Bangalore. RVP thanks UGC (India) for support.
[1] M. Lewenstein, A. Sanpera, V. Ahufinger, Ultracold Atoms in Optical Lattices, (Oxford Univ. Press), (2012).

[2] D. Jaksch, C. Bruder, J. I. Cirac, C. W. Gardiner, and P. Zoller, Phys. Rev. Lett. 81, 3108 (1998).

[3] M. Greiner, O. Mandel, T. Esslinger, T. W. Hänsch, and I. Bloch, Nature 415, 39 (2002).

[4] T. Lahaye, C. Menotti, L. Santos, M. Lewenstein, and T. Pfau, Rep. Prog. Phys., 72, 126401 (2009).

[5] F. Hebert, G. G. Batrouni, R. T. Scalettar, G. Schmid, M. Troyer, and A. Dorneich, Phys. Rev. B 65, 014513 (2001).

[6] G. G. Batrouni, R. T. Scalettar, G. T. Zimanyi, and A. P. Kampf, Phys. Rev. Lett. 74, 2527 (1995).

[7] M. Boninsegni and N. V. Prokof'ev, Phys. Rev. Lett. 95, 237204 (2005).

[8] S. Wessel and M. Troyer, Phys. Rev. Lett. 95, 127205 (2005).

[9] D. Heidarian and K. Damle, Phys. Rev. Lett. 95, 127206 (2005).

[10] R. G. Melko, A. Paramekanti, A. A. Burkov, A. Vishwanath, D. N. Sheng, and L. Balents, Phys. Rev. Lett. 95, 127207 (2005).

[11] G. G. Batrouni, F. Hebert, and R. T. Scalettar, Phys. Rev. Lett. 97, 087209 (2006).

[12] T. Mishra, R. V. Pai, S. Ramanan, M. S. Luthra, and B. P. Das, Phys. Rev. A 80, 043614 (2009).

[13] T. Mishra, R. V. Pai, and B. P. Das, Phys. Rev. B 81, 024503 (2010).

[14] A. Griesmaier, J. Werner, S. Hensler, J. Stuhler, and T. Pfau, Phys. Rev. Lett. 94, 160401 (2005).

[15] Q. Beaufils, R. Chicireanu, T. Zanon, B. Laburthe-Tolra, E. Marechal, L. Vernac, J. C. Keller, and O. Gorceix, Phys. Rev. A 77, 061601 (2008).

[16] M. Lu, N. Q. Burdick, S. H. Youn, and B. L. Lev, Phys. Rev. Lett. 107, 190401 (2011); M. Lu, N. Q. Burdick, and B. L. Lev, ibid. 108, 215301 (2012).

[17] K. Aikawa, A. Frisch, M. Mark, S. Baier, A. Rietzler, R. Grimm, and F. Ferlaino, Phys. Rev. Lett. 108, 210401 (2012).

[18] K.-K. Ni et al., Science 322, 231 (2008); M. H. G. de Miranda et al., Nat. Phys. 7, 502 (2011); A. Chotia, B. Neyenhuis, S. A. Moses, B. Yan, J. P. Covey, M. FossFeig, A. M. Rey, D. S. Jin, and J. Ye, Phys. Rev. Lett. 108, 080405 (2012).
[19] T. F. Gallagher and P. Pillet, in Advances in Atomic, Molecular, and Optical Physics, edited by E. Arimondo (Academic Press, London, 2008), Vol. 56, p. 161.

[20] M. A. Cazalilla, R. Citro, T. Giamarchi, E. Orignac, and M. Rigol, Reviews of Modern Physics 83, 1405 (2011).

[21] T. Giamarchi, Quantum Physics in One Dimension, (Oxford Univ. Press), (2006).

[22] E. Dagotto and T. M. Rice, Science 271, 618 (1996).

[23] M. Uehara, T. Nagata, J. Akimitsu, H. Takahashi, N. Mri1 and K. Kinoshita, J. Phys. Soc. Jpn. 65, 2764 (1996).

[24] C. Kim, A. Y. Matsuura, Z.-X. Shen, N. Motoyama, H. Eisaki, S. Uchida, T. Tohyama, and S. Maekawa, Phys. Rev. Lett. 77, 4054 (1996).

[25] M. A. Cazalilla, A. F. Ho and T. Giamarchi, New J. Phys. 8, 158 (2006).

[26] P. Donohue and T. Giamarchi, Phys. Rev. B 63, 180508(R) (2001).

[27] M. S. Luthra, T. Mishra, R. V. Pai, and B. P. Das, Phys. Rev. B 78, 165104 (2008).

[28] E. Orignac and T. Giamarchi, Phys. Rev. B 64, 144515 (2001).

[29] A. Dhar, M. Maji, T. Mishra, R. V. Pai, S. Mukerjee, and A. Paramekanti, Phys. Rev. A 85, 041602(R) (2012).

[30] A. Dhar, T. Mishra, M. Maji, R. V. Pai, S. Mukerjee, and A. Paramekanti, Phys. Rev. B 87, 174501 (2013).

[31] A. Dhar, T. Mishra, R. V. Pai, S. Mukerjee, and B. P. Das, Phys. Rev. A 88, 053625 (2013).

[32] T. Mishra, R. V. Pai, and S. Mukerjee, Phys. Rev. A 89, 013615 (2014).

[33] T. Mishra, R. V. Pai, S. Mukerjee, and Arun Paramekanti, Phys. Rev. B 87, 17450 (2013).

[34] A. Petrescu and K. Le Hur, Phys. Rev. Lett. 111, 150601 (2013).

[35] M. P. Zaletel, S. A. Parameswaran, A. Rüegg and E. Altman, arXiv:1308.3237 (2013).

[36] W. S. Cole, S. Zhang, A. Paramekanti, and N. Trivedi, Phys. Rev. Lett. 109, 085302 (2012).

[37] M. Dalmonte, G. Pupillo, and P. Zoller, Phys. Rev. Lett. 105, 140401 (2010).

[38] M. Bauer and Meera M. Parish, Phys. Rev. Lett. 108, 255302 (2012).

[39] I. Danshita, J. E. Williams, C. A. R. Sá de Melo, and C. W. Clark, Phys. Rev. A 76, 043606 (2007). 
[40] I. Danshita, Carlos A. R. Sá de Melo, and Charles W. Clark, Phys. Rev. A 77, 063609 (2008).

[41] M. Albiez, R. Gati, J. Fölling, S. Hunsmann, M. Cristiani, and M. K. Oberthaler, Phys. Rev. Lett. 95, 010402 (2005).

[42] M. Atala, M. Aidelsburger, M. Lohse, J. T. Barreiro, B. Paredes, I. Bloch, arXiv:1402.0819

[43] A. J. Daley, J. M. Taylor, S. Diehl, M. Baranov, and P. Zoller, Phys. Rev. Lett. 102, 040402 (2009).

[44] K. P. Schmidt, J. Dorier, A. Läuchli, and F. Mila, Phys. Rev. B 74, 174508 (2006).

[45] M. W. J. Romans, R. A. Duine, Subir Sachdev, and H. T. C. Stoof, Phys. Rev. Lett. 93, 020405 (2004).

[46] L. Bonnes and S. Wessel, Phys. Rev. Lett. 106, 185302 (2011).

[47] Y.-W. Lee and M.-F. Yang, Phys. Rev. A 81, 061604(R) (2010).

[48] Y.-C. Chen, K.-K. Ng, and M.-F. Yang, Phys. Rev. B 84, 092503 (2011).

[49] A. J. Daley and J. Simon, arXiv:1311.1783v1

[50] S. Greschner, L. Santos, and T. Vekua, Phys. Rev. A 87, 033609 (2013).

[51] S. R. White, Phys. Rev. Lett. 69, 2863 (1992).

[52] U. Schollwöck, Rev. Mod. Phys. 77, 259 (2005).
[53] D. van Oosten, P. van der Straten, and H. T. C. Stoof, Phys. Rev. A 63, 053601 (2001).

[54] K. Sheshadri, H. R. Krishnamurthy, R. Pandit, and T. V. Ramakrishnan, Europhys. Lett. 22, 257 (1993).

[55] Ramesh V. Pai, K. Sheshadri, and R. Pandit, Phys. Rev. B 77, 014503 (2008).

[56] R. V. Pai, J. M. Kurdestany, K. Sheshadri, and R. Pandit, Phys. Rev. B 85, 214524 (2012).

[57] M. Singh, A. Dhar, T. Mishra, R. V. Pai, and B. P. Das, Phys. Rev. A 85, 051604 (R).

[58] A. Dhar, M. Singh, R. V. Pai, B. P. Das, Phys. Rev. A 84, 033631 (2011).

[59] P. Buonsante, V. Penna, and A. Vezzani, Laser Physics, 15, 361 (2005).

[60] T. McIntosh, P. Pisarski, R. J. Gooding, and E. Zaremba, Phys. Rev. A 86, 013623 (2012).

[61] D. Huerga, J. Dukelsky, and G. E. Scuseria, Phys. Rev. Lett. 111, 045701 (2013).

[62] D. Yamamoto, A. Masaki, and I. Danshita, Phys. Rev. B 86, 054516 (2012).

[63] S. R. Hassan and L. de' Medici, Phys. Rev. B 81, 035106 (2010).

[64] J. Carrasquilla, F. Becca, and M. Fabrizio, Phys. Rev. B 83, 245101 (2011).

[65] F. Crépin, N. Laflorencie, G. Roux, and P. Simon, Phys. Rev. B 84, 054517 (2011). 